\title{
Trimethoprim-sulfamethoxazole and risk of sudden death among patients taking spironolactone
}

\author{
Tony Antoniou PhD, Simon Hollands MSc, Erin M. Macdonald MSc, Tara Gomes MHSc, \\ Muhammad M. Mamdani PharmD MPH, David N. Juurlink MD PhD; for the Canadian Drug Safety \\ and Effectiveness Research Network
}

Competing interests:

Muhammad Mamdani has served on advisory boards for AstraZeneca, BristolMyers Squibb, Eli Lilly,

GlaxoSmithKline, Hoffman-La Roche, Novartis, Novo Nordisk and Pfizer. No other competing interests were declared.

This article has been peer reviewed.

\section{Correspondence to:}

Tony Antoniou,

tantoniou@smh.ca

CMAJ 2015. DOI:10.1503 /cmaj.140816

\begin{abstract}
- Abstract
Background: Trimethoprim-sulfamethoxazole increases the risk of hyperkalemia when used with spironolactone. We examined whether this drug combination is associated with an increased risk of sudden death, a consequence of severe hyperkalemia.

Methods: We conducted a population-based nested case-control study involving Ontario residents aged 66 years or older who received spironolactone between Apr. 1, 1994, and Dec. 31, 2011. Within this group, we identified cases as patients who died of sudden death within 14 days after receiving a prescription for trimethoprimsulfamethoxazole or one of the other study antibiotics (amoxicillin, ciprofloxacin, norfloxacin or nitrofurantoin). For each case, we identified up to 4 controls matched by age and sex. We determined the odds ratio (OR) for the association between sudden death and exposure to each antibiotic relative to amoxicillin, adjusted for predictors of sudden death using a disease risk index.
\end{abstract}

Results: Of the 11968 patients who died of sudden death while receiving spironolactone, we identified 328 whose death occurred within 14 days after antibiotic exposure. Compared with amoxicillin, trimethoprimsulfamethoxazole was associated with a more than twofold increase in the risk of sudden death (adjusted OR 2.46, 95\% confidence interval [Cl] 1.55-3.90). Ciprofloxacin (adjusted OR 1.55, 95\% Cl 1.02-2.38) and nitrofurantoin (adjusted OR 1.70, 95\% Cl 1.03-2.79) were also associated with an increased risk of sudden death, although the risk with nitrofurantoin was not apparent in a sensitivity analysis.

Interpretation: The antibiotic trimethoprimsulfamethoxazole was associated with an increased risk of sudden death among older patients taking spironolactone. When clinically appropriate, alternative antibiotics should be considered in these patients.
$\mathrm{T}$ he use of spironolactone increased considerably following publication of the Randomized Aldactone Evaluation Study, which showed that the drug improved morbidity and mortality in carefully selected patients with severe systolic heart failure. ${ }^{1,2}$ Although spironolactone is generally well tolerated, hyperkalemia is a potentially life-threatening adverse effect of the drug in clinical practice. ${ }^{3-5}$ Strategies for mitigating the risk of serious hyperkalemia include cautious dosing of spironolactone, close monitoring of electrolyte levels and avoidance of other drugs that cause hyperkalemia.

The widely used antibiotic trimethoprim has pharmacologic similarities to the potassium-sparing diuretic amiloride. It reduces urinary potassium excretion by about $40 \%$ and can increase the risk of life-threatening hyperkalemia in susceptible individuals, including those taking spironolactone. ${ }^{6,7}$ In combination with sulfamethox- azole, trimethoprim is most often used for the treatment of urinary tract infections. More than 20 million prescriptions are written for the combination each year in the United States. ${ }^{8}$

We have previously shown that the use of trimethoprim-sulfamethoxazole in patients receiving spironolactone increased the risk of hospital admission with hyperkalemia more than 12-fold relative to amoxicillin. ${ }^{9}$ However, we did not examine whether the drug interaction was associated with an increased risk of sudden cardiac death, a predictable consequence of severe hyperkalemia. ${ }^{10,11}$ This is important because sudden death in patients taking spironolactone may erroneously be attributed to intrinsic heart disease.

Because treatment with trimethoprimsulfamethoxazole can precipitate life-threatening hyperkalemia in patients receiving spironolactone, we conducted a study to determine whether 
this drug interaction would be associated with an increased risk of sudden death.

\section{Methods}

\section{Setting}

We conducted a population-based, nested casecontrol study involving patients aged 66 years or older living in the province of Ontario who received spironolactone between Apr. 1, 1994, and Dec. 31, 2011. These individuals have universal access to physician services and hospital care and have provincial prescription drug coverage.

\section{Data sources}

We identified prescription drug records using the Ontario Drug Benefit Database, which contains comprehensive records of prescription drugs dispensed to residents in the province aged 65 years or older. We obtained hospital admission data from the Canadian Institute for Health Information's Discharge Abstract Database, which contains detailed clinical information on all hospital admissions in Ontario. We used the Ontario Health Insurance Plan database to identify claims for physician services, and we used validated disease registries to define the presence of diabetes mellitus, hypertension, HIV infection and congestive heart failure. ${ }^{12-15}$ We obtained basic demographic data from the Registered Persons Database, a registry of all residents of the province who are eligible for health insurance. We determined emergency department visits using the $\mathrm{Na}$ tional Ambulatory Care Reporting System. We ascertained sudden deaths from the Ontario Office of the Registrar General's database, which contains the cause of death reported on individual death certificates. These datasets were linked with the use of unique, encoded identifiers and analyzed at the Institute for Clinical Evaluative Sciences, and are routinely used to study the consequences of drug interactions., ${ }^{9}, 16-18$

\section{Study population}

We used the Ontario Drug Benefit Database to identify patients who were prescribed spironolactone between Apr. 1, 1994, and Dec. 31, 2011. For each patient, we defined a period of continuous spironolactone use beginning with the first prescription following their 66th birthday. We excluded the first year of eligibility for prescription drug coverage (age 65) to avoid incomplete medication records. Observation ended with the first occurrence of death, the end of the study period or the cessation of spironolactone treatment, which was defined as a lapse of more than 100 days between successive prescriptions. In the event of such a lapse, we extended the observation period 100 days from the date of the last prescription to identify outcomes that might have precipitated cessation of therapy. We used prescription intervals of 100 days to define continuous use of spironolactone, because this is the maximum prescription interval allowed by the provincial drug benefit program.

Within the cohort of continuous users of spironolactone, we defined cases as patients who died of sudden death within 14 days after receiving a prescription for one of trimethoprimsulfamethoxazole, ciprofloxacin, norfloxacin, nitrofurantoin or amoxicillin (excluding amoxicillin-clavulinic acid). The date of death served as the index date for all analyses. We excluded patients who received prescriptions for more than one of the antibiotics of interest, or any other antibiotic in the 30 days before the index date. We also excluded patients admitted to hospital within 30 days before the index date, individuals with a history of HIV infection or organ transplantation, and individuals who filled prescriptions for oral corticosteroids or immunomodulating drugs in the 100 days before the index date, to avoid the confounding effects of recent serious illness and immune compromise. We identified sudden death using a previously validated case definition, which has a positive predictive value of $86.8 \% .^{19}$

From within the cohort of patients receiving spironolactone, we selected up to 4 controls for each case matched by age at the index date (within $1 \mathrm{yr}$ ) and sex. Controls were required to be alive at the index date, and to have received one of the study antibiotics within 14 days before the index date. We excluded controls who received prescriptions for any other antibiotic in the 30 days before the index date. Consequently, all cases and controls were older patients receiving spironolactone who did not receive any antibiotic in the 30 days before the index date other than one of the study antibiotics. When fewer than 4 controls were available for each case, we included only those controls and maintained the matching process. We excluded cases that could not be matched to at least 1 control.

\section{Statistical analysis}

We used standardized differences to compare baseline demographic and clinical characteristics of cases and controls. Standardized differences of less than 0.1 indicate good balance between the cases and controls for a given covariate..$^{20}$

We used conditional logistic regression to estimate the odds ratio (OR) and $95 \%$ confidence intervals (CIs) for the association between sudden death and receipt of a prescription for trimethoprim-sulfamethoxazole, with patients receiving amoxicillin as the reference group. We selected 
amoxicillin as the reference antibiotic because it does not interact with spironolactone and is not associated with sudden death.

To contextualize our findings, we conducted similar analyses for norfloxacin, nitrofurantoin and

Table 1: Characteristics of patients taking spironolactone who experienced sudden death within 14 days after receiving co-prescription of trimethoprimsulfamethoxazole or another study antibiotic* (cases) and matched controls

\begin{tabular}{|c|c|c|c|}
\hline \multirow[b]{2}{*}{ Variable } & \multicolumn{2}{|c|}{ No. (\%) of patientst } & \multirow[b]{2}{*}{$\begin{array}{l}\text { Standardized } \\
\text { difference }\end{array}$} \\
\hline & $\begin{array}{c}\text { Cases } \\
n=328\end{array}$ & $\begin{array}{l}\text { Controls } \\
n=1171\end{array}$ & \\
\hline Age, yr, median (IQR) & $86(81-90)$ & $86(81-90)$ & 0.07 \\
\hline \multicolumn{4}{|l|}{ Age group, yr } \\
\hline $66-74$ & $34(10.4)$ & $123(10.5)$ & 0.01 \\
\hline $75-84$ & $84(25.6)$ & $332(28.4)$ & 0.06 \\
\hline$\geq 85$ & $210(64.0)$ & $716(61.1)$ & 0.07 \\
\hline Female sex & $217(66.2)$ & $807(68.9)$ & 0.06 \\
\hline $\begin{array}{l}\text { Duration of spironolactone use, } \\
\text { yr, median (IQR) }\end{array}$ & $1(1-2)$ & $3(1-5)$ & 0.66 \\
\hline \multicolumn{4}{|l|}{ Charlson Comorbidity Index } \\
\hline No hospital admission & $56(17.1)$ & $389(33.2)$ & 0.36 \\
\hline 0 & $20(6.1)$ & $128(10.9)$ & 0.16 \\
\hline 1 & $55(16.8)$ & $201(17.2)$ & 0.01 \\
\hline$\geq 2$ & $197(60.1)$ & $453(38.7)$ & 0.44 \\
\hline \multicolumn{4}{|l|}{ Comorbid condition } \\
\hline Congestive heart failure & $258(78.7)$ & $681(58.2)$ & 0.43 \\
\hline Angina§ & $7 \quad(2.1)$ & $30(2.6)$ & 0.03 \\
\hline Acute myocardial infarction§ & $14(4.3)$ & $33(2.8)$ & 0.08 \\
\hline Dysrhythmia§ & $135(41.2)$ & $370(31.6)$ & 0.20 \\
\hline Chronic kidney disease§ & $9(2.7)$ & $30(2.6)$ & 0.01 \\
\hline Diabetes mellitus & $113(34.5)$ & $359(30.7)$ & 0.08 \\
\hline Hypertension & 249 (75.9) & $892(76.2)$ & 0.01 \\
\hline $\begin{array}{l}\text { Residence in long-term care } \\
\text { facility }\end{array}$ & $201(61.3)$ & $445(38.0)$ & 0.48 \\
\hline $\begin{array}{l}\text { No. of prescription drugs in } \\
\text { previous year, median (IQR) }\end{array}$ & $18(14-22)$ & $15(12-19)$ & 0.37 \\
\hline \multicolumn{4}{|l|}{ Medication use in preceding $90 \mathrm{~d}$} \\
\hline Non-potassium-sparing diuretic & $284(86.6)$ & $833(71.1)$ & 0.36 \\
\hline Potassium-sparing diuretic $\mathbb{1}$ & $\leq 5$ & $\leq 5$ & 0.00 \\
\hline$\beta$-Adrenergic receptor antagonist & $114(34.8)$ & $400(34.2)$ & 0.01 \\
\hline Potassium supplement & $11(3.4)$ & $37(3.2)$ & 0.01 \\
\hline NSAID & $42(12.8)$ & $155(13.2)$ & 0.01 \\
\hline $\begin{array}{l}\text { Renin-angiotensin-aldosterone } \\
\text { inhibitor }\end{array}$ & $176(53.7)$ & $524(44.7)$ & 0.18 \\
\hline \multicolumn{4}{|l|}{ Income quintile } \\
\hline 1 (lowest) & $83(25.3)$ & $284(24.3)$ & 0.02 \\
\hline 2 & $71(21.6)$ & $239(20.4)$ & 0.03 \\
\hline 3 & $56(17.1)$ & $238(20.3)$ & 0.08 \\
\hline 4 & $56(17.1)$ & $227(19.4)$ & 0.06 \\
\hline 5 (highest) & $58(17.7)$ & $177(15.1)$ & 0.07 \\
\hline \multicolumn{4}{|c|}{$\begin{array}{l}\text { Note: IQR = interquartile range, NSAID = nonsteroidal anti-inflammatory drug. } \\
\text { ^Amoxicillin, ciprofloxacin, norfloxacin or nitrofurantoin. } \\
\text { tUnless stated otherwise. } \\
\text { ‡Difference between cases and controls divided by standard deviation. } \\
\text { Sln past } 3 \text { years. } \\
\text { ๆOther than spironolactone; prevalence not reported because of small cell size. }\end{array}$} \\
\hline
\end{tabular}

ciprofloxacin, because these drugs are commonly used for the treatment of urinary tract infections. ${ }^{21}$ We anticipated no association between sudden death and norfloxacin, because it has no direct cardiac effects and does not interact with spironolactone. In contrast, we anticipated an association with ciprofloxacin and with nitrofurantoin, because the former tends to be used for more serious infections and can independently cause prolongation of the QT interval, ${ }^{22,23}$ and the latter was associated with an increased risk of hyperkalemia in patients receiving spironolactone in our earlier study. ${ }^{9}$

To prevent model overfitting, we adjusted our analysis for an extensive array of covariates associated with sudden death using a disease risk index. ${ }^{24}$ We derived the disease risk index for each patient using the $\beta$ coefficients obtained from a nonparsimonious multivariable logistic regression model that included sudden cardiac death as the dependent variable and an extensive list of medical conditions and classes of prescription drugs related to the risk of this outcome (Appendix 1, available at www.cmaj.ca/lookup /suppl/doi:10.1503/cmaj.140816/-/DC1). The disease risk index was then introduced as a single term in the conditional logistic regression model along with indicators for each antibiotic and duration of spironolactone use. To test the robustness of our findings, we repeated the analysis by matching cases and controls by age, sex and disease risk index (within 0.2 standard deviations). Because the disease risk index was developed specifically for the purposes of this study, it has not been previously validated.

To provide an approximation of the absolute risk of sudden death associated with antibiotic use, we determined the number of sudden deaths occurring within 14 days after receiving each antibiotic relative to the total number of prescriptions for each drug in the same subset of patients.

We performed all analyses using SAS version 9.3 (SAS Institute).

\section{Ethics approval}

The study design was approved by the Research Ethics Board of the Sunnybrook Health Sciences Centre, Toronto.

\section{Results}

During the 17-year study period, we identified 206319 patients who received treatment with spironolactone. Of these, 11968 experienced sudden death, including 349 who died within 14 days after receiving one of the study antibiotics. Of the 349 patients, $328(94.0 \%)$ were matched to at least 1 control. Most of the patients were aged 85 years or older, and as expected, 
comorbidities and medications associated with sudden death were more prevalent among the cases than among the controls (Table 1). The majority of spironolactone prescriptions dispensed to cases (95\%) and controls (94\%) were for the 25-mg formulation of the drug.

In the main analysis, sudden death during spironolactone treatment was more than twice as likely following a prescription for trimethoprimsulfamethoxazole than for amoxicillin (adjusted OR 2.46, 95\% CI 1.55-3.90) (Table 2). Treatment with ciprofloxacin (adjusted OR 1.55, 95\% CI 1.02-2.38) and nitrofurantoin (adjusted OR $1.70,95 \%$ CI $1.03-2.79)$ were also associated with an increased risk of sudden death during spironolactone therapy. In contrast, we found no such risk with norfloxacin (adjusted OR 0.86, 95\% CI 0.47-1.58).

In the sensitivity analysis, we matched 148 cases to 231 controls, achieving adequate balance of nearly all covariates evaluated (Appendix 3, available at www.cmaj.ca/lookup/suppl /doi:10.1503/cmaj.140816/-/DC1). Estimates for the association between sudden death and trimethoprim-sulfamethoxazole (adjusted OR $1.94,95 \%$ CI $0.93-4.04)$ and ciprofloxacin (adjusted OR 1.40, 95\% CI 0.73-2.67) were attenuated, but they were consistent with a heightened risk of sudden death (Appendix 4, available at www.cmaj.ca/lookup/suppl/doi :10.1503/cmaj.140816/-/DC1). In contrast, an association with nitrofurantoin was not found in the sensitivity analysis (adjusted OR 0.90, 95\% CI 0.43-1.90).

Overall, 29141 courses of trimethoprimsulfamethoxazole in patients receiving spironolactone were associated with 215 deaths within 14 days after exposure, for a rate of $0.74 \%$. The respective rates of death for amoxicillin, ciprofloxacin, norfloxacin and nitrofurantoin were $0.35 \%, 0.54 \%, 0.31 \%$ and $0.39 \%$.

\section{Interpretation}

In this population-based study involving older patients receiving spironolactone treatment, we found that trimethoprim-sulfamethoxazole use was associated with a higher risk of sudden death relative to other antibiotics with similar indications. As expected, treatment with ciprofloxacin was also associated with an increased risk of sudden death among these patients, whereas no such risk was observed with norfloxacin. Our findings were consistent in a sensitivity analysis in which cases and controls were matched by their disease risk index; however, as expected, the results were less precise because of the marked reduction in sample size associated with the matching process for the sensitivity analysis. Although we observed an association between sudden death and nitrofurantoin in the main analysis, this finding was no longer apparent in the sensitivity analysis. The reasons for this difference are unclear; however, a pharmacodynamic interaction between nitrofurantoin and spironolactone is biologically plausible, because the former may suppress aldosterone levels. ${ }^{25}$

Our study has important clinical implications. Trimethoprim-sulfamethoxazole and spironolactone are commonly prescribed medications, and the likelihood of co-prescription is high. Moreover, fatal hyperkalemia resulting from a drug interaction with trimethoprim-sulfamethoxazole is not likely to be implicated as the cause of death, particularly among older patients taking spironolactone, most of whom have intrinsic heart disease. The risks of this drug interaction can be minimized or avoided through the use of alternate antibiotics, careful patient selection and monitoring, and limited duration of antibiotic treatment, when clinically appropriate.

We speculate that the heightened risk of sudden death associated with trimethoprim-

Table 2: Association between sudden death and recent antibiotic use*

\begin{tabular}{|c|c|c|c|c|}
\hline \multirow[b]{2}{*}{ Antibiotic } & \multicolumn{2}{|c|}{ No. $(\%)$ of patients } & \multicolumn{2}{|c|}{ OR $(95 \% \mathrm{Cl})$} \\
\hline & $\begin{array}{c}\text { Cases } \\
n=328\end{array}$ & $\begin{array}{l}\text { Controls } \\
n=1171\end{array}$ & Unadjusted & Adjusted $\dagger$ \\
\hline TMP/SMX & $86(26.2)$ & $189(16.1)$ & $2.45(1.67-3.61)$ & $2.46(1.55-3.90)$ \\
\hline Nitrofurantoin & 49 (14.9) & $202(17.3)$ & $1.32(0.86-2.02)$ & $1.70(1.03-2.79)$ \\
\hline Norfloxacin & $27 \quad(8.2)$ & $162(13.8)$ & $0.84(0.51-0.39)$ & $0.86(0.47-1.58)$ \\
\hline Ciprofloxacin & $105(32.0)$ & $289(24.7)$ & $1.99(1.38-2.87)$ & $1.55(1.02-2.38)$ \\
\hline Amoxicillin & $61(18.6)$ & $329(28.1)$ & 1.00 (ref) & 1.00 (ref) \\
\hline \multicolumn{5}{|c|}{$\begin{array}{l}\text { Note: } \mathrm{Cl}=\text { confidence interval, } \mathrm{OR}=\text { odds ratio, ref = reference group, TMP/SMX = trimethoprim-sulfamethoxazole. } \\
\text { *Antibiotic use in preceding } 14 \mathrm{~d} \text {. } \\
\text { †Adjusted for disease risk index (covariates in Appendix 2, available at www.cmaj.ca/lookup/suppl/doi:10.1503/cmaj.140816/-I } \\
\text { DC1) and duration of spironolactone use. }\end{array}$} \\
\hline
\end{tabular}


sulfamethoxazole reflects terminal hyperkalemia resulting from its established drug interaction with spironolactone. This reasoning is supported by case reports, clinical trials and our previous research that showed a 12 -fold increased risk of hospital admission with hyperkalemia associated with the same drug combination. ${ }^{9,26-29}$ A similar interaction likely accounts for the increased risk of hyperkalemia and sudden death associated with trimethoprim-sulfamethoxazole among patients receiving renin-angiotensin inhibitors. ${ }^{18,30}$ The mechanism by which trimethoprim can precipitate hyperkalemia in patients receiving spironolactone relates to an amiloride-like inhibition of sodium channels in the luminal membrane of the distal tubule, which results in impaired potassium secretion and sodium reabsorption. ${ }^{31}$

Similarly, the heightened risk of sudden death with nitrofurantoin observed in our main analysis is consistent with our earlier findings of a more than twofold increased risk of hyperkalemia when the drug is combined with spironolactone. ${ }^{9}$ This finding may reflect a selection bias, whereby patients at high risk of sudden death are preferentially prescribed nitrofurantoin rather than trimethoprim-sulfamethoxazole. Alternatively, some data suggest that nitrofurantoin dramatically reduces aldosterone from the adrenal cortex,$^{25}$ which implies a mechanistically different interaction between spironolactone and nitrofurantoin that predisposes susceptible patients to hyperkalemia and sudden death. However, as noted earlier, the association between nitrofurantoin and sudden death should be interpreted in light of the discrepant findings of our sensitivity analysis. In the absence of research confirming the safety of nitrofurantoin in patients receiving spironolactone, selection of an alternative antibiotic should be considered for these patients. Although ciprofloxacin has no known drug interaction with spironolactone, we postulate that the risk of sudden death associated with this drug may be explained by its proarrhythmic properties, variation in treatment indication or its use in patients with particularly severe urinary tract infections.

\section{Limitations}

Some limitations of our work merit emphasis. We used administrative data and thus had no access to the patients' serum potassium levels, indices of renal function, whether antibiotic doses were adjusted for renal function or medication adherence. Although we used a validated algorithm to define sudden death, misclassification is possible. However, these limitations apply equally to all antibiotics. Importantly, we had no information regarding the indications for antibiotic use. Although nitrofurantoin and norfloxacin are limited to the treatment of uncomplicated urinary tract infections, amoxicillin, trimethoprimsulfamethoxazole and ciprofloxacin may be used for other infections. In addition, our findings may not apply to younger patients, who generally have fewer risks for severe hyperkalemia and sudden death. Case patients had a greater comorbidity burden relative to controls. However, this is expected among patients who experience sudden death, and we adjusted for differences between the groups with a disease risk index derived using an extensive array of disease- and drug-related determinants of sudden cardiac death. Furthermore, as with all observational studies, confounding due to unmeasured variables is a potential source of bias. Finally, confounding by illness severity is a potential source of bias, because patients receiving trimethoprim-sulfamethoxazole may be systematically less well than those receiving amoxicillin. However, this is an unlikely explanation for our findings, because the risk of sudden death with ciprofloxacin was lower than that with trimethoprim-sulfamethoxazole, yet ciprofloxacin is generally used to treat more severe or more complicated urinary tract infections.

\section{Conclusion}

We found that trimethoprim-sulfamethoxazole was associated with a marked increase in the risk of sudden death among older patients receiving spironolactone, a finding that we speculate reflects trimethoprim-induced hyperkalemia. We also found a less pronounced but clinically important association with ciprofloxacin, and possibly nitrofurantoin. When clinically appropriate, clinicians should consider alternative antibiotics for patients receiving spironolactone.

\section{References}

1. Pitt B, Zannad F, Remme WJ, et al. The effect of spironolactone on morbidity and mortality in patients with severe heart failure. N Engl J Med 1999;341:709-17.

2. Masoudi FA, Gross CP, Wang Y, et al. Adoption of spironolactone therapy for older patients with heart failure and left ventricular systolic dysfunction in the United States, 1998-2001. Circulation 2005;112:39-47.

3. Cruz CS, Cruz AA, Marcílio de Souza CA. Hyperkalemia in congestive heart failure patients using ACE inhibitors and spironolactone. Nephrol Dial Transplant 2003;18:1814-9.

4. Svensson M, Gustafsson F, Galatius S, et al. How prevalent is hyperkalemia and renal dysfunction during treatment with spironolactone in patients with congestive heart failure? J Card Fail 2004;10:297-303.

5. Bozkurt B, Agoston I, Knowlton AA. Complications of inappropriate use of spironolactone in heart failure: when an old medicine spirals out of new guidelines. J Am Coll Cardiol 2003; 41:211-4.

6. Eiam-Ong S, Kurtzman NA, Sabatini S. Studies on the mechanism of trimethoprim-induced hyperkalemia. Kidney Int 1996; 49:1372-8.

7. Velázquez H, Perazella MA, Wright FS, et al. Renal mechanism of trimethoprim-induced hyperkalemia. Ann Intern Med 1993; 119:296-301. 
8. Hicks LA, Taylor TH Jr. US outpatient antibiotic prescribing, 2010. N Engl J Med 2013;368:1461-2.

9. Antoniou T, Gomes T, Mamdani MM, et al. Trimethoprimsulfamethoxazole induced hyperkalemia in elderly patients receiving spironolactone: nested case-control study. BMJ 2011 343:d5228.

10. McIntyre WF, Femenía F, Arce M, et al. Importance of early electrocardiographic recognition and timely management of hyperkalemia in geriatric patients. Exp Clin Cardiol 2011;16:47-50.

11. Sood MM, Sood AR, Richardson R. Emergency management and commonly encountered outpatient scenarios in patients with hyperkalemia. Mayo Clin Proc 2007;82:1553-61.

12. Hux JE, Ivis F, Flintoft V, et al. Diabetes in Ontario: determination of prevalence and incidence using a validated administrative data algorithm. Diabetes Care 2002;25:512-6.

13. Tu K, Campbell NR, Chen ZL, et al. Accuracy of administrative databases in identifying patients with hypertension. Open Med 2007;1:e18-26.

14. Antoniou T, Zagorski B, Loutfy MR, et al. Validation of casefinding algorithms derived from administrative data for identifying adults living with human immunodeficiency virus infection. PLOS ONE 2011;6:e21748.

15. Schultz SE, Rothwell DM, Chen Z, et al. Identifying cases of congestive heart failure from administrative data: a validation study using primary care patient records. Chronic Dis Inj Can 2013;33:160-6.

16. Juurlink DN, Mamdani M, Kopp A, et al. Drug-drug interactions among elderly patients hospitalized for drug toxicity. JAMA 2003;289:1652-8.

17. Antoniou T, Gomes T, Mamdani MM, et al. Trimethoprimsulfamethoxazole-induced phenytoin toxicity in the elderly: a population-based study. Br J Clin Pharmacol 2011;71:544-9.

18. Antoniou T, Gomes T, Juurlink DN, et al. Trimethoprimsulfamethoxazole-induced hyperkalemia in patients receiving inhibitors of the renin-angiotensin system: a population-based study. Arch Intern Med 2010;170:1045-9.

19. Chung CP, Murray KT, Stein CM, et al. A computer case definition for sudden cardiac death. Pharmacoepidemiol Drug Saf 2010;19:563-72.

20. Austin PC, Grootendorst P, Anderson GM. A comparison of the ability of different propensity score models to balance measured variables between treated and untreated subjects: a Monte Carlo study. Stat Med 2007;26:734-53.

21. Anti-infective Review Panel. Anti-infective guidelines for community-acquired infections. Toronto: MUMS Guideline Clearinghouse; 2010.

22. Knorr JP, Moshfeghi M, Sokoloski MC. Ciprofloxacin-induced Q-T interval prolongation. Am J Health Syst Pharm 2008;65: 547-51.

23. Milberg P, Hilker E, Ramtin S, et al. Proarrhythmia as a class effect of quinolones: increased dispersion of repolarization and triangulation of action potential predict torsades de pointes. J Cardiovasc Electrophysiol 2007;18:647-54.

24. Arbogast PG, Kaltenbach L, Ding H, et al. Adjustment for multiple cardiovascular risk factors using a summary risk score. Epidemiology 2008;19:30-7.

25. Jager LP, de Graaf GJ, Widjaja-Greefkes HCA. Differential ef fects of nitrofurans on the production/release of steroid hormones by porcine adrenocortical cells in vitro. Eur J Pharmacol 1997;331:325-31.
26. Marinella MA. Severe hyperkalemia associated with trimethoprim-sulfamethoxazole and spironolactone. Infect Dis Clin Pract 1997;6:257.

27. Martin J, Mourton S, Nicholls G. Severe hyperkalemia with prescription of potassium-retaining agents in an elderly patient. $N Z$ Med J 2003;116:U542.

28. Alappan R, Buller GK, Perazella MA. Trimethoprimsulfamethoxazole therapy in outpatients: Is hyperkalemia a sig nificant problem? Am J Nephrol 1999;19:389-94.

29. Perazella MA, Mahnensmith RL. Trimethoprim-sulfamethoxazole: hyperkalemia is an important complication regardless of dose. Clin Nephrol 1996;46:187-92.

30. Fralick M, Macdonald EM, Gomes T, et al. Co-trimoxazole and sudden death in patients receiving inhibitors of reninangiotensin system: population based study. BMJ 2014;349: g6196.

31. Velázquez H, Perazella MA, Wright FS, et al. Renal mechanism of trimethoprim-induced hyperkalemia. Ann Intern Med 1993 119:296-301.

Affiliations: Department of Family and Community Medicine (Antoniou), Li Ka Shing Knowledge Institute (Antoniou, Gomes, Mamdani), St. Michael's Hospital; University of Toronto (Gomes, Mamdani, Juurlink); Institute for Clinical Evaluative Sciences (Antoniou, Hollands, Macdonald, Gomes, Mamdani, Juurlink); Sunnybrook Research Institute (Juurlink), Toronto, Ont.

Contributors: All of the authors conceived and designed the study. Simon Hollands acquired the data. All of the authors analyzed and interpreted the data. Tony Antoniou drafted the manuscript. All of the authors critically revised the manuscript for important intellectual content, approved the final version of the manuscript submitted for publication and agreed to act as guarantors of the work.

Funding: Tony Antoniou is supported by a New Investigator Award from the Canadian Institutes of Health Research. This project was supported by research funds from the Canadian Drug Safety and Effectiveness Research Network and by the Institute for Clinical Evaluative Sciences, which is funded by a grant from the Ontario Ministry of Health and Long-Term Care. The sponsors had no role in the design and conduct of the study; in the collection, analysis and interpretation of the data; or in the preparation, review and approval of the manuscript. The opinions, results and conclusions reported in this paper are those of the authors and are independent from the funding sources. No endorsement by the Institute for Clinical Evaluative Sciences or the Ontario Ministry of Health and Long-Term Care is intended or should be inferred.

Acknowledgement: The authors thank IMS Brogan Inc. for use of its Drug Product and Therapeutic Class Database to access the drug identification numbers used to identify study drugs in the Ontario Drug Benefit Database. 